\title{
Challenges and Management of Surgical Site Occurrences
}

\author{
Moalla Muzzafar ${ }^{1} \quad$ Humara Noreen $^{2} \quad$ Muhammad Ahmad Raouf $^{3}$ \\ 1.Resident Plastic Surgery, Alnoor Hospital, Makkah KSA \\ 2.Woman Medical Officer RHC JamanShah, Layyah \\ 3.Medical Officer BHU Kothay Wala, Multan
}

\begin{abstract}
Summary
Postoperative complications such as surgical site infections, dehiscence, seromas, and hematomas prolong wound care and impose significant cost increases to patients and healthcare providers. Clinicians aiming to reduce the incidence of these complications should be aware of risk factors associated with surgical type, procedures used, patient characteristics, and postoperative care. Today, improved guidelines and general practices for managing surgical incisions have reduced the incidence of complications to historic lows. In addition to these standard care options, advanced wound care approaches have been extensively studied and exist as options for clinicians to provide adjunctive postoperative support and facilitate wound healing. These systems include advanced wound dressings and closed-incision negative-pressure therapy. Advanced wound care is not appropriate in all settings, and healthcare providers must assess each case for specific needs to be addressed by the available incision management plans. Emerging therapies that are intended to improve the continuum of postoperative care should continue to be evaluated in controlled clinical trials to determine their effectiveness under different circumstances and to support the creation of more robust guidelines for their use.
\end{abstract}

DOI: $10.7176 / \mathrm{JMPB} / 53-04$

Publication date:March $31^{\text {st }} 2019$

\section{Introduction}

Postoperative complications following the 45 million operations performed each year in the United States result in substantial morbidity and costs.1 Common complications include surgical site infections (SSIs), dehiscences, and development of seromas or hematomas. These complications delay hospital discharge, prolong wound care, and lead to the need for additional invasive procedures. The increased morbidity and costs associated with complications motivate healthcare providers to be vigilant in recognizing risk factors arising from patient comorbidities or circumstances of surgery. Current clinical research continues to provide evidence for new techniques that aim at reducing the incidence of these complications.

\section{COMMON SURGICAL SITE OCCURRENCES.}

Among postoperative complications, SSIs are one of the most prevalent, accounting for $21.8 \%$ of the $\approx 721,800$ healthcare-associated infections reported annually.2 Each incident increases the average hospital stay by 9.6 days at an additional cost of $\$ 38,656$, which results in over $\$ 10$ billion in direct and indirect costs annually.3 In addition to a monetary cost, the presence of an SSI is associated with the occurrence of other complications and a higher 30-day mortality rate.4 Common risk factors associated with SSI are listed in Table 1. Dehiscence of a closed surgical wound is often preceded by the presence of an SSI,6,7 with some medical researchers reporting dehiscence and SSI as interchangeable. However, the exact relationship between SSI and dehiscence is difficult to determine due to the lack of reliable standardized data and discrepant recording methodologies.8 Another common surgical site complication is the formation of seromas or hematomas at the incision site, typically when an anatomical space has been created. In either case, both can increase tension at the incision, making these sites more prone to infection. 9 
Table NO 1.

\begin{tabular}{ll}
\hline Patient-related Risk Factors & Incision-related Risk Factors \\
\hline - Diabetes mellitus & - High-tension incision \\
- ASA score $\geq 3$ & - Repeated incisions \\
- Advanced age & - Extensive undermining \\
- Obesity & - Traumatized soft tissue \\
- Active tobacco use & - Contamination \\
- Hypoalbuminemia & - Emergency procedure \\
- Corticosteroid usage & - Mechanically unfavorable site \\
- Active alcoholism & - Prolonged operation time \\
- Male sex & - Postsurgical radiation \\
- Chronic renal & \\
- insufficiency & \\
- Chronic obstructive & \\
- Hemolmaration disease &
\end{tabular}

\section{SURGERY-SPECIFIC RISK FACTORS.}

Risks of surgery have been related to various factors including the location, type, and surgical duration. Patients receiving emergency surgery or complex operations have a higher than average risk of experiencing SSIs.4 Increased duration of operations is also associated with elevated number of surgical site occurrences. Among surgery types, specific procedures can also hold higher risk of SSI than others (Table 2). The rate of SSI within 30 days of surgery ranges 3\%-4\% for integumentary, musculoskeletal, respiratory, and oral surgeries, while the rate is slightly higher $(4 \%-5 \%)$ for surgical resolution of vascular abnormalities. 4 Abdominal surgeries have the highest 30-day rate of SSIs, at over 6\%. Hernia repair overall maintains a low 30-day SSI rate of 55 years of age, wound infection, emergency surgery, and steroid use.6,13

\section{MANAGEMENT OF SURGICAL SITE OCCURRENCES.}

Common practices for managing postoperative incisions typically include placement of a nonadherent or silverimpregnated material on the incision, followed by sterile gauze or abdominal pads.14 Incidence of surgical complications has been reduced by improvement in operating room ventilation, instrument sterilization procedures, maximum barrier protection requirements, and the use of prophylactic antibiotics. Preoperative cleaning of the surgical site with antiseptic solutions is another common practice with the intention of reducing SSIs; however, multiple meta-analyses have found sparse evidence demonstrating any benefit against SSI incidence. 15,16

\section{ADVANCED WOUND CARE.}

In addition to the first-line treatment practices, there have been attempts by medical researchers to identify other advanced wound care strategies that might reduce the risk of postoperative complications. One approach includes the application of antimicrobial advanced wound dressings (AWDs) over the closed incision. In a single-center retrospective review of 903 patients with total joint arthroplasty, a silver-releasing, cellulose-based antimicrobial AWD was more effective than conventional dressings in reducing the incidence of periprosthetic joint infections.17 In a prospective, randomized controlled trial (RCT) of a different silver-eluting antimicrobial AWD used postoperatively in patients after lower extremity arterial reconstruction, intervention with AWD failed to show a significant benefit reducing wound complications.18 Another technology that has been used to enhance postsurgery wound healing is closed-incision negative-pressure therapy (ciNPT), which creates a closed wound environment and applies negative pressure using a foam dressing. Highquality studies have also reported benefits of ciNPT in postoperative complications. An RCT involving 102 patients with groin incisions found that those treated with ciNPT had a significantly shorter mean duration of hospital stay compared (6 days) with a control group (9 days).19 A second RCT of 100 patients with 129 groin incisions reported that patients treated with ciNPT exhibited a significant reduction in 30-day wound infections ( $2.3 \%$ versus $17.5 \%$, respectively) and revision surgeries (1.7\% versus $14.1 \%$, respectively) compared to patients who received standard care. 20 
Table No 2.

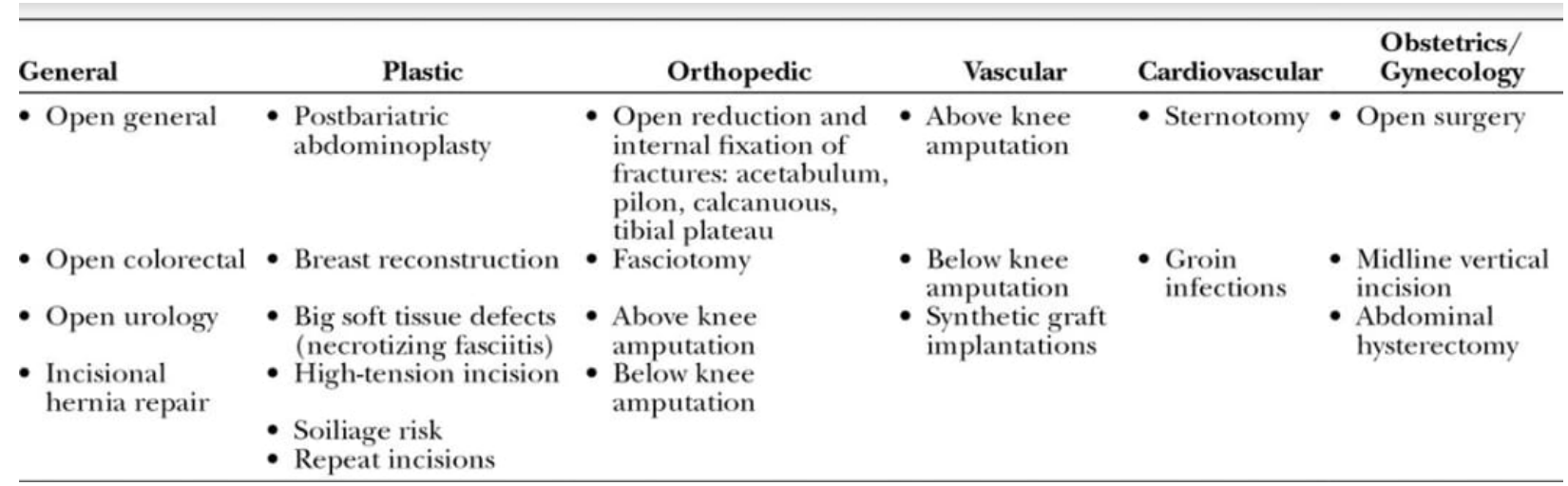

In an RCT investigating postcesarean wound morbidity in 82 patients, patients treated with ciNPT had fewer surgical site occurrences $(5.1 \%)$ than a control group receiving standard care $(16.3 \%)$. Patients with ciNPT also experienced reduced incisional pressure (54.3\% versus $91.3 \%$, respectively). 21 A 2015 meta-analysis of 8 controlled studies involving a total of 1,277 patients found that ciNPT provided a significant reduction in SSI risk. However, there was no significant impact of ciNPT on risk of dehiscence and hematoma.22 In a retrospective analysis of 138 revision hip and knee operations comparing a silver-releasing, cellulose-based AWD to ciNPT, the ciNPT group exhibited a significantly reduced number of complications in general, and SSIs specifically.23 It is important to note that not all surgical incisions may benefit from ciNPT. In an RCT following 81 patients at high risk of surgical site complications with multiple comorbidities after lower extremity and abdominal surgery, there was no significant difference in SSI or dehiscence between patient groups treated with dry dressings compared to those receiving ciNPT.24 Therefore, attention should be paid to specific characteristics of the patient and surgery procedure to determine whether the patient would benefit from ciNPT. ciNPT has been rapidly introduced into clinical medicine, and we are still learning which patients will best benefit from this, and trying to better understand the mechanisms of action. Surgeons have proposed a number of mechanisms of action such as relieving tension along the incision, altering the blood flow to the wound, changing the kinetics of lymphangiogenesis, and reducing fluid and infectious materials. The molecular basis for each of these mechanisms continues to be developed.

\section{CONCLUSIONS.}

Surgical site occurrences pose a substantial threat to patient health and can result in costly or invasive interventions, making use of adequate prevention practices a necessity. Healthcare practitioners should assess the patient's risk of postoperative complications by taking into account patient comorbidities, the quality of the surgical site, and the characteristics of the operation itself. Although standard protocols have improved to reduce incidence of surgical site complications, emerging advanced wound care technologies may provide additional benefit in select circumstances. These new therapies must continue to be evaluated to better define their appropriate use and determine improved protocols for decreasing complication risk.

\section{REFERENCES}

1. Hall MJ, DeFrances CJ, Williams SN, et al. National hospital discharge survey: 2007 summary. Natl Health Stat Report. 2010:1-20, 24.

2. Magill SS, Edwards JR, Bamberg W, et al. Multistate pointprevalence survey of health care-associated infections. N Engl J Med. 2014;370:1198-1208.

3. Shepard J, Ward W, Milstone A, et al. Financial impact of surgical site infections on hospitals: the hospital management perspective. JAMA Surg. 2013;148:907-914.

4. Neumayer L, Hosokawa P, Itani K, et al. Multivariable predictors of postoperative surgical site infection after general and vascular surgery: results from the patient safety in surgery study. J Am Coll Surg. 2007;204:1178-1187.

5. Willy C, Agarwal A, Andersen CA, et al. Closed incision negative pressure therapy: international multidisciplinary consensus recommendations. Int Wound J. 2017;14:385-398.

6. Khan MN, Naqvi AH, Irshad K, et al. Frequency and risk factor of abdominal wound dehiscence. J Coll Physicians Surg Pak. 2004;14:355-357.

7. Aksamija G, Mulabdic A, Rasic I, et al. Evaluation of risk factors of surgical wound dehiscence in adults after laparotomy. Med Arch. 2016;70:369-372.

8. Sandy-Hodgetts K, Carville K, Leslie GD. Determining risk factors for surgical wound dehiscence: a literature review. Int Wound J. 2015;12:265-275. 
9. Bullocks J, Basu CB, Hsu P, et al. Prevention of hematomas and seromas. Semin Plast Surg. 2006;20:233-240.

10. Rosen MJ, Bauer JJ, Harmaty M, et al. Multicenter, prospective, longitudinal study of the recurrence, surgical site infection, and quality of life after contaminated ventral hernia repair using biosynthetic absorbable mesh: the COBRA study. Ann Surg. 2017;265:205-211.

11. McCarthy CM, Mehrara BJ, Riedel E, et al. Predicting complications following expander/implant breast reconstruction: an outcomes analysis based on preoperative clinical risk. Plast Reconstr Surg. 2008;121:1886-1892.

12. Beecher SM, O’Leary DP, McLaughlin R, et al. Influence of complications following immediate breast reconstruction on breast cancer recurrence rates. Br J Surg. 2016;103:391-398.

13. Pavlidis TE, Galatianos IN, Papaziogas BT, et al. Complete dehiscence of the abdominal wound and incriminating factors. Eur J Surg. 2001;167:351-354; discussion 355.

14. Nam D, Sershon RA, Levine BR, et al. The use of closed incision negative-pressure wound therapy in orthopaedic surgery. J Am Acad Orthop Surg. 2018;26:295-302.

15. Dumville JC, McFarlane E, Edwards P, et al. Preoperative skin antiseptics for preventing surgical wound infections after clean surgery. Cochrane Database Syst Rev. 2013;3:CD003949.

16. Webster J, Osborne S. Preoperative bathing or showering with skin antiseptics to prevent surgical site infection. Cochrane Database Syst Rev. 2015:CD004985.

17. Cai J, Karam JA, Parvizi J, et al. Aquacel surgical dressing reduces the rate of acute PJI following total joint arthroplasty: a case-control study. J Arthroplasty. 2014;29:1098-1100.

18. Ozaki CK, Hamdan AD, Barshes NR, et al. Prospective, randomized, multi-institutional clinical trial of a silver alginate dressing to reduce lower extremity vascular surgery wound complications. J Vasc Surg. 2015;61:419-427.e1.

19. Lee K, Murphy PB, Ingves MV, et al. Randomized clinical trial of negative pressure wound therapy for highrisk groin wounds in lower extremity revascularization. J Vasc Surg. 2017;66:1814-1819.

20. Pleger SP, Nink N, Elzien M, et al. Reduction of groin wound complications in vascular surgery patients using closed incision negative pressure therapy (ciNPT): a prospective, randomised, single-institution study. Int Wound J. 2018;15:75-83.

21. Gunatilake RP, Swamy GK, Brancazio LR, et al. Closedincision negative-pressure therapy in obese patients undergoing cesarean delivery: a randomized controlled trial. AJP Rep. 2017;7:e151-e157.

22. Sandy-Hodgetts K, Watts R. Effectiveness of negative pressure wound therapy/closed incision management in the prevention of post-surgical wound complications: a systematic review and meta-analysis. JBI Database System Rev Implement Rep. 2015;13:253-303.

23. Cooper HJ, Bas MA. Closed-incision negative-pressure therapy versus antimicrobial dressings after revision hip and knee surgery: a comparative study. J Arthroplasty. 2016;31:1047-1052.

24. Masden D, Goldstein J, Endara M, et al. Negative pressure wound therapy for at-risk surgical closures in patients with multiple comorbidities: a prospective randomized controlled study. Ann Surg. 2012;255:1043-1047. 